\title{
HER2-targeted recombinant protein immuno-caspase-6 effectively induces apoptosis in HER2-overexpressing GBM cells in vitro and in vivo
}

\author{
LEIMING ZHANG $^{1,2 *}$, JUNLIN REN $^{3,4 *}$, HANGYU ZHANG $^{5 *}$, GANG CHENG $^{1}$, YANMING XU $^{4}$, \\ SHUANGWU YANG ${ }^{2}, \mathrm{CHAO} \mathrm{DONG}^{1}$, DANDONG FANG ${ }^{1}$, JIANNING ZHANG ${ }^{1,2}$ and ANGANG YANG ${ }^{4}$ \\ ${ }^{1}$ Department of Neurosurgery, PLA Navy General Hospital, Beijing 100048; ${ }^{2}$ Department of Neurosurgery, Xijing \\ Institute of Clinical Neuroscience, Xijing Hospital, The Fourth Military Medical University, Xi'an, Shaanxi 710032; \\ ${ }^{3}$ Department of Clinical Laboratory, PLA Navy General Hospital, Beijing 100048; ${ }^{4}$ State Key Laboratory of Cancer \\ Biology, Department of Immunology, The Fourth Military Medical University, Xi'an, Shaanxi 710032; ${ }^{5}$ Department of \\ Hepatobiliary Surgery, Xijing Institute of Clinical Neuroscience, Xijing Hospital, Xi'an, Shaanxi 710032, P.R. China
}

Received March 12, 2016; Accepted July 18, 2016

DOI: $10.3892 /$ or.2016.5088

\begin{abstract}
Glioblastoma multiforme (GBM), which is associated with a high rate of morbidity and mortality, is among the most malignant and treatment-refractory neoplasms in human adults. As GBM is highly resistant to conventional therapies, immunotherapies are a promising treatment candidate. HER2 is an attractive target for GBM immunotherapy, as its expression is highly associated with various types of GBM. We previously reported that a novel HER2-targeted recombinant protein e23sFv-Fdt-casp6 has an antitumor effect on HER2-positive gastric cancer cells. In this study, we established a genetically modified Chinese hamster ovary cell line, which produced and secreted e23sFv-Fdt-casp6 proteins. Following specific binding to and internalization into HER2-overexpressing tumor cells, the e23sFv-Fdt-casp6 protein induced tumor cell apoptosis and inhibited the proliferation of HER2overexpressing A172 and U251MG cells in vitro, but not in U87MG cells with undetectable HER2. The e23sFv-Fdt-casp6 gene was introduced into severe combined immunodeficient mice bearing human glioblastoma xenografts by using intramuscular injections of a liposome-encapsulated vector. The
\end{abstract}

Correspondence to: Dr Jianning Zhang, Department of Neurosurgery, PLA Navy General Hospital, 6 Fucheng Road, Beijing 100048, P.R. China

E-mail: jianningzg@sina.com

Dr Angang Yang, State Key Laboratory of Cancer Biology, Department of Immunology, The Fourth Military Medical University, 127 Changle West Road, Xi'an, Shanxi 710032, P.R. China

E-mail: agyang@fmmu.edu.cn

*Contributed equally

Key words: apoptosis, HER2, glioblastoma, immunotherapy recombinant protein e23sFv-Fdt-casp6 specifically targeted tumor cells and induced apoptosis, thereby leading to potent inhibition of tumor growth and prolonged the survival time of tumor-bearing mice. We concluded that e23sFv-Fdt-casp6 represents a promising HER2-targeted treatment option for human gliomas.

\section{Introduction}

Glioblastoma multiforme (GBM) is the most frequently encountered primary central nervous system neoplasm in adults, and is associated with high morbidity and mortality. Despite multimodal treatment options, including surgical resection, radiotherapy, and chemotherapy, the median survival of patients with glioblastoma is $12-15$ months and 2-5 years for patients with anaplastic gliomas (1). GBM arises as a result of various genetic alterations and the deregulation of growth-factor signaling pathways, resulting in abnormal cellular proliferation, malignant differentiation, and increased invasiveness of malignant cells $(2,3)$.

Human epidermal growth factor receptor 2 (HER2) is a cell membrane receptor with tyrosine kinase activity. HER2 plays critical roles in cell proliferation, differentiation, adhesion, and motility (4). The gene amplification and protein overexpression of HER2 in human tumors is associated with a poor prognosis (5). According to previous studies, HER2 is expressed by up to $80 \%$ of GBM cases $(6,7)$. The frequent overexpression of HER2 in GBMs (8-10) and its rare expression in the adult central nervous system (11) make HER2 an ideal therapeutic target in GBMs.

Apoptosis, a process in which cells actively participate in their own death, is often disrupted in tumor cells (12). Therefore, the introduction of proapoptotic molecules may be an effective approach for the treatment of tumors, including malignant gliomas. Caspases, a family of cysteine proteases, play a vital role in transducing apoptosis signals and executing apoptosis in mammalian cells (13). Constitutively active recombinant caspase- 6 is capable of autocatalytic processing in vitro and 
in vivo, and can induce apoptosis independent of endogenous apoptosis signals (14). Therefore recombinant caspase-6 could be used at very low concentrations to induce apoptosis in tumor cells. It has been reported that tumor specific delivery of recombinant caspase- 6 to gliomas triggered apoptosis independent of upstream apoptotic signaling (15). In our previous studies, we generated a novel immuno-caspase-6 (immuno-casp6) containing a HER2-specific single-chain antibody (e23sFv), a furin cleavage sequence from diphtheria toxin (Fdt), and a constitutively active caspase-6 molecule (casp6) (16). Following receptor-mediated endocytosis and furin-mediated cleavage in the endosome, the e $23 \mathrm{sFv}$-Fdt-casp 6 proteins engaged in direct translocation of the released $\mathrm{C}$-terminal fragment (active caspase-6) and induced apoptosis in HER2-overexpressing gastric tumor cells. The purpose of the present study is to investigate the proapoptotic effects of the novel immuno-casp6 (e23sFv-Fdt-casp6) and its therapeautic efficacy in HER2-overexpressing malignant gliomas.

\section{Materials and methods}

Cell culture. The human GBM cell lines A172, U251MG, and U87MG [American Type Culture Collection (ATCC), Manassas, VA, USA], and a Chinese hamster ovary (CHO) cell line (Chinese Academy of Sciences Cell Bank, Shanghai, China) were cultured in Dulbecco's modified Eagle's medium (DMEM) supplemented with $10 \%$ fetal bovine serum (FBS) (both from Gibco, Grand Island, NY, USA) at $37^{\circ} \mathrm{C}$ in $5 \% \mathrm{CO}_{2}$.

PCR amplification. Genomic DNA was extracted from $\mathrm{CHO}$ cells that were stably transfected with an empty pCMV vector or pCMV-e23sFv-Fdt-casp6, after G418 selection, by using 5'-TTT GCG GCC GCG AAA GCC GGC AAT AGA GTG AGG AGA TCT GTG GGC GCA GCC TCC GTT TAC-3' as the upstream primer and 5'-TTT TCT AGA TTA ATC TAC TAC ATC CAA AGG AAT-3' as the downstream primer. A fusion gene that encompassed the sequence encoding Fdt and active caspase- 6 was amplified from the genomic templates.

Western blot analysis. CHO culture supernatants were concentrated by centrifugation for $30 \mathrm{~min}$ at $4^{\circ} \mathrm{C}$ and $3,000 \mathrm{x} \mathrm{g}$ in Amicon Ultra concentrators (30,000 MWCO; Millipore Corp., Billerica, MA, USA). Phosphate-buffered saline (PBS) was added to the device, and centrifugation was performed again as above to concentrate the supernatant and change the buffers (17). The proteins from the whole-cell lysate and concentrated supernatants were blotted onto PVDF membranes. The membranes were then incubated overnight at $4^{\circ} \mathrm{C}$ with primary antibodies against active caspase-6 (1:500; Abcam, Cambridge, UK), HER2 (1:500; NeoMarkers, Fremont, CA, USA), and then incubated with horseradish peroxidase-conjugated secondary antibody (1:2,000; Zhongshan Golden Bridge Biotechnology Co., Beijing, China) for $2 \mathrm{~h}$ at room temperature. The blots were visualized using an enhanced chemiluminescence kit (Pierce Biotechnology, Inc., Rockford, IL, USA).

Expression of HER 2 examined by flow cytometry. Cells from the human GBM cell lines A172, U251MG, and U87MG were washed with PBS, blocked with rabbit serum, and then incubated with mouse anti-HER2 antibody (1:100; NeoMarkers) for $30 \mathrm{~min}$. The appropriate isotype antibody was used as a negative control. Cells were extensively washed with PBS and incubated with PE-conjugated rabbit anti-mouse IgG (1:200; BD Biosciences, San Diego, CA, USA) for $30 \mathrm{~min}$. Finally, samples were analyzed via flow cytometry (Becton-Dickinson, San Diego, CA, USA).

Immunofluorescent staining. Stably transfected $\mathrm{CHO}$ cells were cultured on cover slips in DMEM containing $10 \%$ FBS and then fixed in a freshly prepared $4 \%$ paraformaldehyde solution for $10 \mathrm{~min}$ at room temperature. Then, they were permeabilized with $0.1 \%$ Triton $\mathrm{X}-100$ for $10 \mathrm{~min}$, and incubated overnight at $4^{\circ} \mathrm{C}$ with rabbit anti-active caspase- 6 (1:200; Abcam). Cells were washed extensively with PBS and incubated with Cy3-labelled goat anti-rabbit secondary antibodies (1:100; Boster Inc., Wuhan, China) for $30 \mathrm{~min}$ at room temperature followed by further rinsing. DAPI (Invitrogen, Carslbad, CA, USA) was used for nuclear staining. Finally, cells were washed with PBS, mounted on slides, and observed under a fluorescence microscope (Olympus, Tokyo, Japan).

A172, U251MG, and U87MG cells were cultured on cover slips. The supernatant was aspirated and replaced with freshly collected medium from stably transfected $\mathrm{CHO}$ cell cultures every 24 h. Immunofluorescent staining was carried out 2 days later, as described above. DAPI was used for nuclear staining. The cells were observed under a fluorescence microscope (Olympus).

Analysis of apoptosis by flow cytometry. A172, U251MG, and U87MG cells were cultured for 2 days with freshly collected culture supernatant from stably transfected CHO cells. The medium was aspirated and replaced every $24 \mathrm{~h}$. After washing twice with ice-cold PBS, the cells were stained with FITC-labeled Annexin V staining solution and propidium iodide (PI) (Roche, Basel, Switzerland) according to the manufacturer's instructions and analyzed using a flow cytometer.

Cell proliferation assay. GBM cells A172, U251MG, and U87MG were cultured in 96-well plates at a density of $5 \times 10^{3}$ cells/well with freshly collected culture supernatant from stably transfected $\mathrm{CHO}$ cells. The medium was aspirated and replaced every $24 \mathrm{~h}$. The cells were then incubated with $20 \mu \mathrm{l}$ of $1.5 \mathrm{mg} / \mathrm{ml}$ MTT per well for $4 \mathrm{~h}$, followed by the addition of $150 \mathrm{ml}$ DMSO at each indicated time-point. A490 nm values were determined using a Sunrise microplate reader (Tecan Austria GmbH, Salzburg, Austria). Each assay was performed in triplicate on at least 3 independent occasions.

In vivo antitumor activity of the e23sFv-Fdt-casp6 protein. Severe combined immunodeficient (SCID) mice aged 6-7 weeks were purchased from the Vital River Laboratory Animal Technology Co. (Beijing, China) and were cared for and used in compliance with the guidelines of Animal Center of the Fourth Military Medical University. The mice were inoculated subcutaneously with $5 \times 10^{6} \mathrm{U} 251 \mathrm{MG}$ cells in the right posterior limb. When tumors reached a diameter of 5-7 mm (day 0), mice were randomly assigned to 2 groups, 15 mice/group. The mice in each group were further randomly classified into 2 subgroups, in which 7 mice were used for immunohistochemical staining and the TUNEL assay, and 8 mice 
for survival analysis. The treatment group received 6 doses of $10 \mu \mathrm{g}$ Lipofectamine-encapsulated pCMV-e23sFv-Fdt-casp6 every 3 days by intramuscular injection into the right posterior limb. Control mice were injected with Lipofectamine mixed with the empty pCMV vector. Tumor growth was monitored using a caliper to measure 2 perpendicular tumor diameters every 4 days. The volume of the tumor was then calculated using the formula: tumor volume $=($ width $) 2 \times$ (length $/ 2)$. On day 28 mice were sacrificed by cervical dislocation, and dissected to collect organ tissues. Tissues were embedded in paraffin for further analysis. For the survival analysis, the mice received the same treatment as before and their survival time was recorded.

Distribution of e23sFv-Fdt-casp6 via immunohistochemical analysis. Tissues were fixed in $10 \%$ formalin and embedded in paraffin, sectioned at $4 \mu \mathrm{m}$ thickness, then dewaxed and blocked with $0.3 \% \mathrm{H}_{2} \mathrm{O}_{2}$. The sections were incubated with trypsin and normal goat serum, followed by rabbit anti-active caspase-6 (1:50; Abcam). The appropriate isotype antibody was used as a negative control. Secondary biotinylated goat anti-rabbit IgG antibody (Dako, Glostrup, Denmark) was added, followed by streptavidin-horseradish peroxidase (Sigma-Aldrich, St. Louis, MO, USA). The slides were stained with diaminobenzidine (Sigma-Aldrich) and counterstained with hematoxylin.

TUNEL assay. DNA fragmentation was detected using the TUNEL assay. The TUNEL assay was performed according to the manufacturer's instructions (Roche). DAPI was used for nuclei staining. The cells were observed under a fluorescent microscope (Olympus).

Statistical analysis. All data were analyzed using SPSS v19 and are presented as means \pm SD of at least 3 independent experiments. Student's t-test was used to analyze the difference between groups. For the in vivo experiments, the survival times were analyzed using the Kaplan-Meier method and the log-rank test was used to assess the difference between groups. A value of $\mathrm{P}<0.05$ was considered statistically significant.

\section{Results}

Expression of HER2 in GBM cell lines. HER2 is expressed by up to $80 \%$ of GBMs $(6,7)$. To gain further information on the expression status of HER2 in GBM cell lines, we used western blot analysis and flow cytometry analyses to determine the protein expression of HER2 in A172, U251MG, and U87MG. High levels of HER2 protein were detected in the A172 and U251MG cells, and low expression was observed in U87MG cells (Fig. 1A). HER2 expression on the cell surface of A172, U251MG, and U87MG cells was detected via flow cytometry, and percent of cells expressing HER2 was 36.5, 21.5 and 3.3\%, respectively (Fig. 1B). Based on these results, in subsequent experiments A172 and U251MG cell lines were chosen for in vitro and in vivo studies, whereas U87MG was employed as a negative control.

Expression and secretion of the recombinant protein in $\mathrm{CHO}$ cells. The immuno-casp6 gene was cloned downstream and in
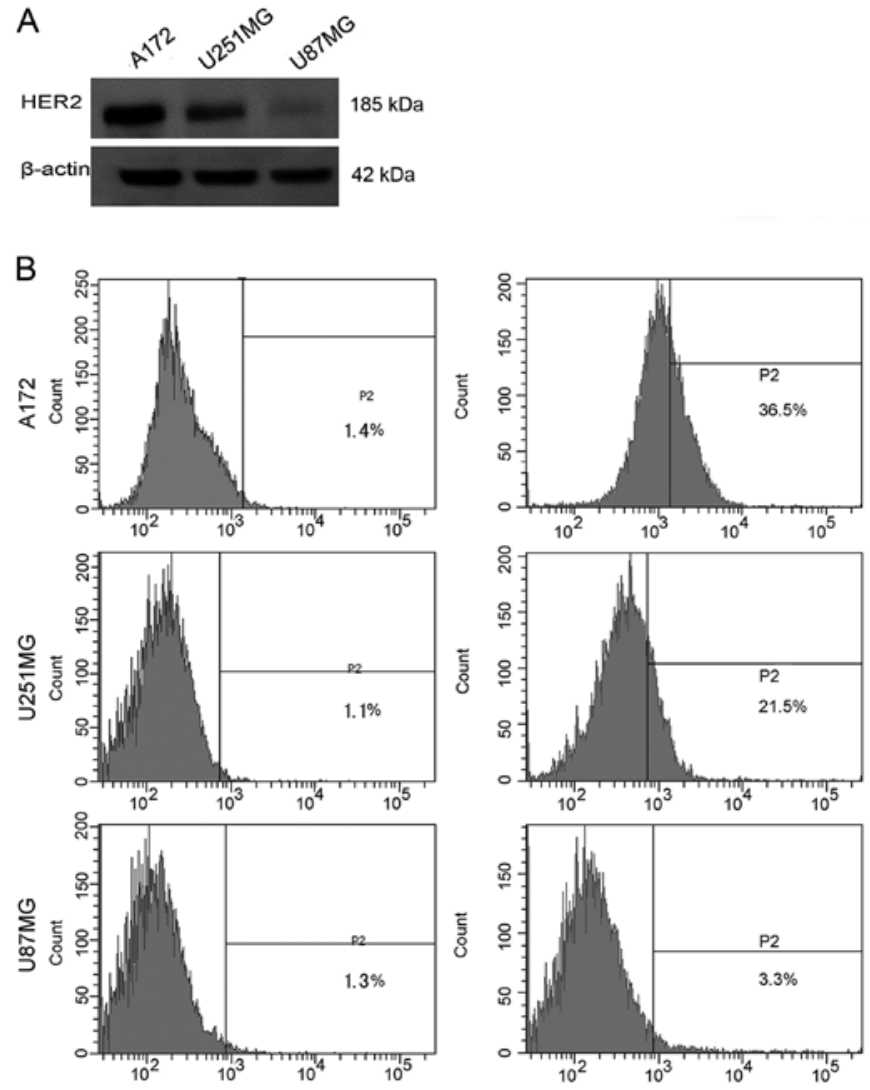

Figure 1. Expression of HER2 in glioblastoma multiforme (GBM) cell lines detected by western blot analysis and flow cytometry. (A) Western blot analysis was performed on the A172, U251MG, and U87MG GBM cell lines as indicated. HER 2 was detected at $185 \mathrm{kDa}$ and was present in the A172 and U251MG cells, but not in U87MG. $\beta$-actin was used as an internal control for equal protein loading. (B) Flow cytometry results showing percentage of cells expressing HER2 in A172, U251MG, and U87MG cells.

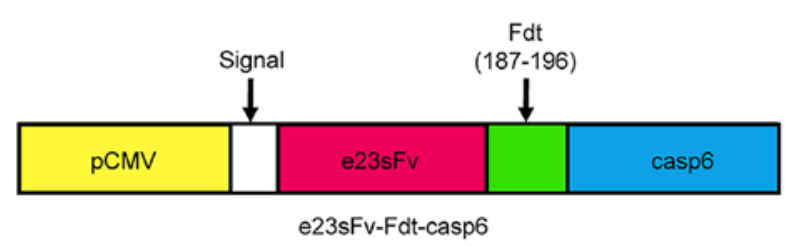

Figure 2. Schematic diagram of the composition of the chimeric molecule pCMV-e23sFv-Fdt-casp6. A single peptide was added to the N-terminal of e23sFv-Fdt-casp6 to facilitate the secretion of the fusion protein; e23sFv, an anti-human epithelial receptor type 2 (anti-HER2) single chain antibody fragment; Fdt, a short furin cleavage sequence from the diphtheria toxin translocation domain; casp6, a constitutively active caspase-6.

frame with the DNA encoding a signal sequence in the expression vector pCMV (Fig. 2). CHO cells are important host cells for the industrial production of pharmaceutical proteins owing to their capacity for correct folding, assembly, and post-translational modification of recombinant proteins (18). To obtain continuous and high yields of the recombinant proteins, $\mathrm{CHO}$ cells were stably transfected with the empty pCMV or the pCMV-e23sFv-Fdt-casp6 vector and selected for with G418. The chimeric gene was specifically amplified from the transduced cells for genomic PCR analysis (Fig. 3A), indicating that the e23sFv-Fdt-casp6 gene was integrated into the host $\mathrm{CHO}$ cell's genome. The expression of the e23sFv-Fdt-casp6 protein 


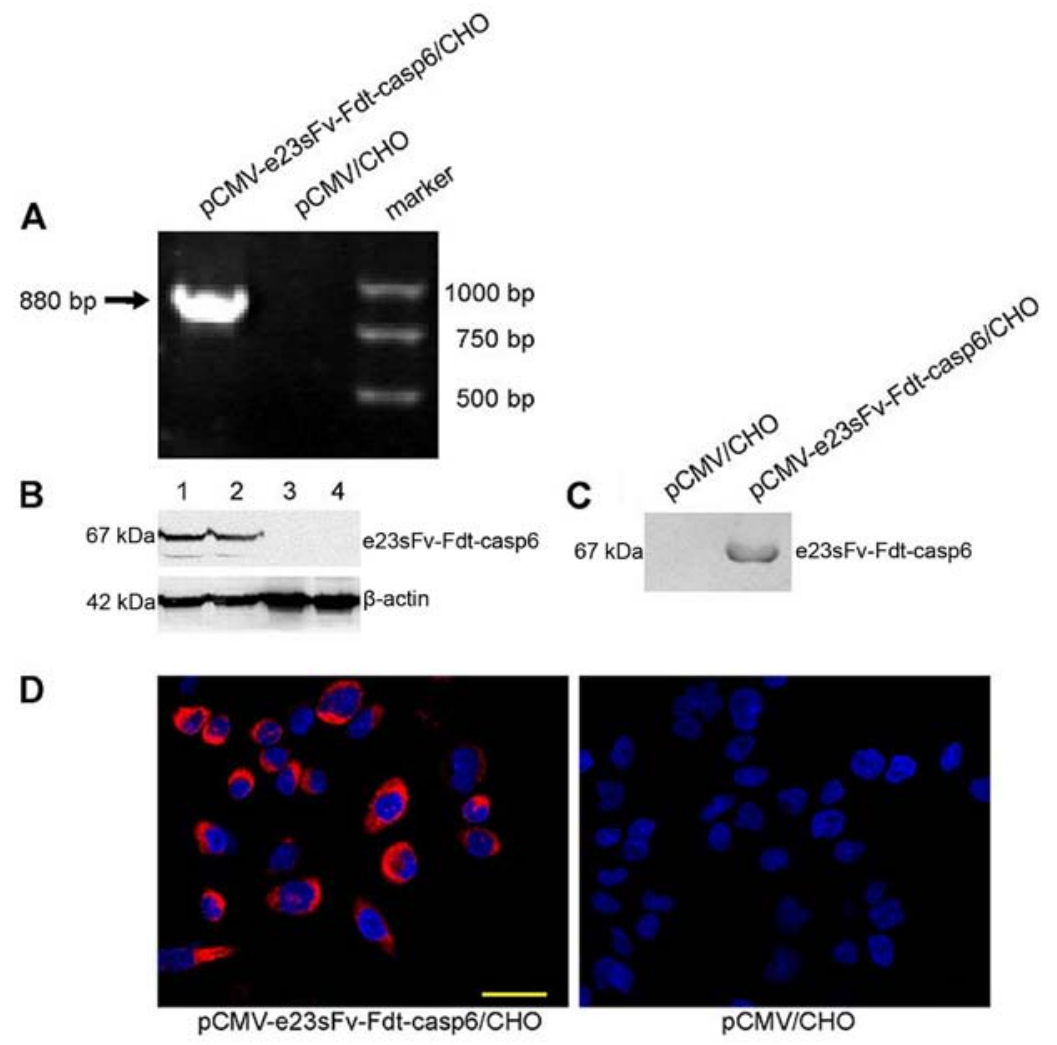

Figure 3. Expression and secretion of the fusion protein from genetically modified Chinese hamster ovary (CHO) cells. Control pCMV plasmids or recombinant plasmids containing e23sFv-Fdt-casp6 expression cassettes were stably transduced into CHO cells. (A) An 880 bp fragment of the Fdt-casp6 fusion gene was amplified from the genomic DNA of the $\mathrm{CHO}$ cells genetically modified with e23sFv-Fdt-casp6, but not from that of the control cells. (B) Western blotting was performed on selected colonies to examine the expression of the e23sFv-Fdt-caps6 protein by the CHO cells using an anti-caspase- 6 antibody as a probe. Lanes 1 and 2, colonies containing pCMV-e23sFv-Fdt-caps6; lanes 3 and 4, control colonies containing pCMV only. (C) Western blotting was performed to determine the protein expression of e23sFv-Fdt-casp6 from the culture supernatant, using an anti-caspase-6 antibody as a probe. (D) Detection of the expression of the fusion protein in genetically modified $\mathrm{CHO}$ cells using indirect immunofluorescence staining with rabbit anti-active caspase- 6 and cy3-labelled goat anti-rabbit secondary antibodies (red). Cells were counterstained with the nuclear stain DAPI (blue). Magnification, x400.

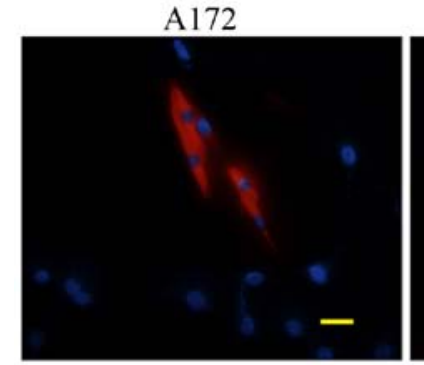

Red --- Caspase-6/cy-3

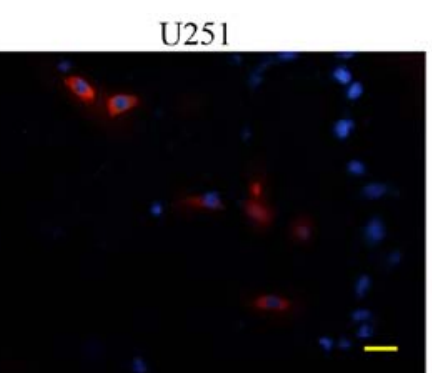

Blue --- DAPI

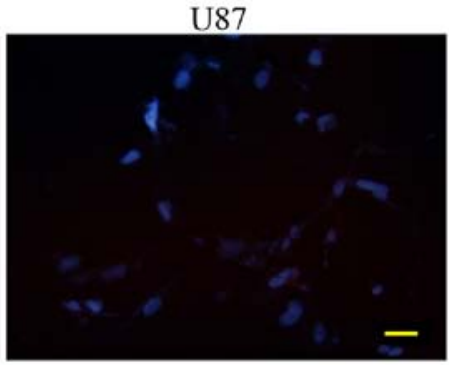

Figure 4. Specific binding and internalization of the e23sFv-Fdt-casp6 fusion protein into HER2-positive GBM cells was confirmed using immunofluorescence staining. After being cultured in conditioned medium from the CHO cells expressing the fusion protein, some A172 and U251MG cells showed positive immunofluorescence staining for active caspase-6 (red) but no staining could be observed in U87MG cells. The cell nuclei were counterstained with DAPI (blue). Magnification, $\mathrm{x} 400$.

was confirmed via western blot analysis both in the transfected $\mathrm{CHO}$ cells and in the cell culture supernatant (Fig. 3B and C). Furthermore, the recombinant proteins were stained strongly in the cytoplasm of the stably transfected CHO cells (Fig. 3D), indicating a high expression efficiency of the recombinant protein in the modified $\mathrm{CHO}$ cells. The $\mathrm{CHO}$ cells producing the e23sFv-Fdt-casp6 protein showed a normal morphology and proliferation relative to the parental CHO cells (Fig. 3D), suggesting that they did not experience toxicity from the fusion proteins.
Binding and internalization of e23sFv-Fdt-casp6 in HER2-overexpressing GBM cells. To confirm whether the secreted e23sFv-Fdt-casp6 recombinant protein can specifically bind to and be subsequently internalized by HER2-overexpressing GBM cells, we cultured A172, U251MG, and U87MG cells with freshly collected supernatants from the modified $\mathrm{CHO}$ cells. After 2 days of incubation, e23sFv-Fdt-casp6 recombinant proteins produced by the modified CHO cells were concentrated in many of the A172 and U251MG cells, but not in the HER2-negative U87MG 

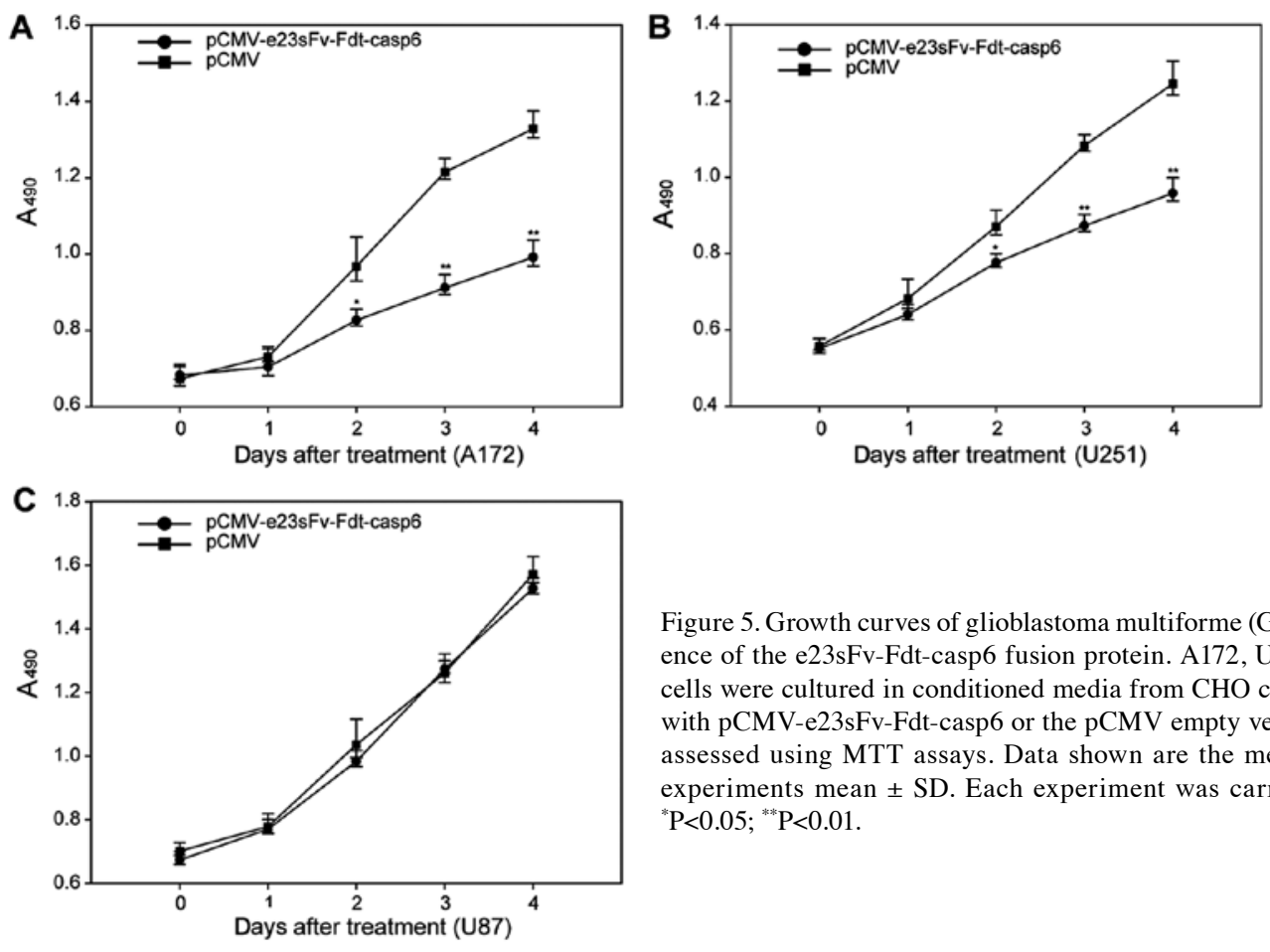

Figure 5. Growth curves of glioblastoma multiforme (GBM) cells in the presence of the e23sFv-Fdt-casp6 fusion protein. A172, U251MG, and U87MG cells were cultured in conditioned media from $\mathrm{CHO}$ cells stably transfected with $\mathrm{pCMV}$-e23sFv-Fdt-casp6 or the pCMV empty vector. Cell growth was assessed using MTT assays. Data shown are the mean of 3 independent experiments mean $\pm \mathrm{SD}$. Each experiment was carried out in triplicate. ${ }^{*} \mathrm{P}<0.05 ;{ }^{* *} \mathrm{P}<0.01$.

cells, indicating the specific binding and internalization of the recombinant protein by the HER2-overexpressing GBM cells (Fig. 4).

Proliferation inhibition, and apoptosis induction in HER2-overexpressing GBM cells bye23sFv-Fdt-casp6. A172, U251MG, and U87MG cells were cultured with supernatants from the modified $\mathrm{CHO}$ cells. Cells were cultured with supernatants from the normal $\mathrm{CHO}$ cells as the negative control. After $48 \mathrm{~h}$ of culture, abundant cell death was observed in the A172 and U251MG cells. The MTT assay showed inhibition of proliferation in the A172 and U251MG cells. By contrast, no difference in proliferation was observed in the U87MG cells, suggesting that the inhibition of proliferation depends on the overexpression of HER2 on cell surfaces (Fig. 5). To investigate whether the inhibition of proliferation was induced by cell apoptosis, flow cytometry was performed in A172, U251MG, and U87MG cells cultured with the supernatants of stably transfected CHO cells. After $48 \mathrm{~h}$, Annexin V-FITC staining revealed that $36 \%$ and $21.3 \%$ of A172 and U251MG cells respectively were apoptotic, higher than that in the control cells. No obvious difference was observed in the U87MG cells (Fig. 6).

Tumor growth suppression in SCID mice and distribution of e23sFv-Fdt-casp6 in xenograft tissues. The in vivo therapeutic effect of the fusion protein e23sFv-Fdt-casp6 was evaluated in SCID mice bearing human glioblastoma xenografts. After the establishment of glioblastoma xenografts, the mice were randomly assigned to treatment and control treatment groups, with each mouse receiving 6 doses of $10 \mu \mathrm{g}$ of Lipofectamine-encapsulated pCMV-e23sFv-Fdt-casp6 or pCMV plasmid, respectively. Plasmids were injected intramuscularly in the right posterior limb every 3 days. The tumor growth and survival rates were

monitored and analyzed. The tumors in mice receiving the Lipofectamine-pCMV-e23sFv-Fdt-casp6 complex grew more slowly than those in the control mice, suggesting that the e23sFv-Fdt-casp6 protein secreted by the genetically modified muscle cells suppressed the growth of HER2-positive GBM cells. The average tumor size in mice receiving the Lipofectamine-pCMV-e23sFv-Fdt-casp6 complex at the time they were sacrificed was approximately two third the size of the tumors in the control mice $(544.3 \pm 42.5$ vs. $888.7 \pm 44.2 \mathrm{~mm}^{3}, \mathrm{P}<0.05$ ) (Fig. 7A). The mice treated with the pCMV-e23sFv-Fdt-casp6 construct exhibited a prolonged mean survival time compared with those treated with the control vector ( $72.4 \pm 4.9$ vs. $47.0 \pm 3.1$ days, $\mathrm{P}<0.01$ ) (Fig. $7 \mathrm{~B}$ ).

The distribution of the e23sFv-Fdt-casp6 recombinant proteins was determined in tumor tissues and normal tissues via immunohistochemistry. Positive staining for caspase-6 was observed in the tumor tissues from the treatment mice but not in those from control (Fig. 8A and B). No positive staining was observed in the lung, kidney, brain, liver, or heart tissues of mice treated with the Lipofectamine-pCMV-e23sFv-Fdtcasp6 complex (data not shown). Therefore, e23sFv-Fdt-casp6 protein produced and secreted by the modified muscle cells specifically localizes to HER2-positive tumor cells. Similarly, in the TUNEL assay, an abundance of apoptotic cells were detected in tumors from mice treated with the Lipofectamine -pCMV-e23sFv-Fdt-casp6 complex, but not in the tumors from the control mice treated with the Lipofectamine-pCMV complex (Fig. 8C-F). Hematoxylin and eosin staining was performed to evaluate the toxicity of the recombinant protein on other important organs, such as lung, kidney, brain, liver, and heart. We found no signs of massive cell injury (Fig. 8G-K), which suggested the recombinant protein e23sFv-Fdt-casp6 can effectively target and induce apoptosis in HER2-overexpressing tumor cells with no obvious side-effects to normal tissues. 
A
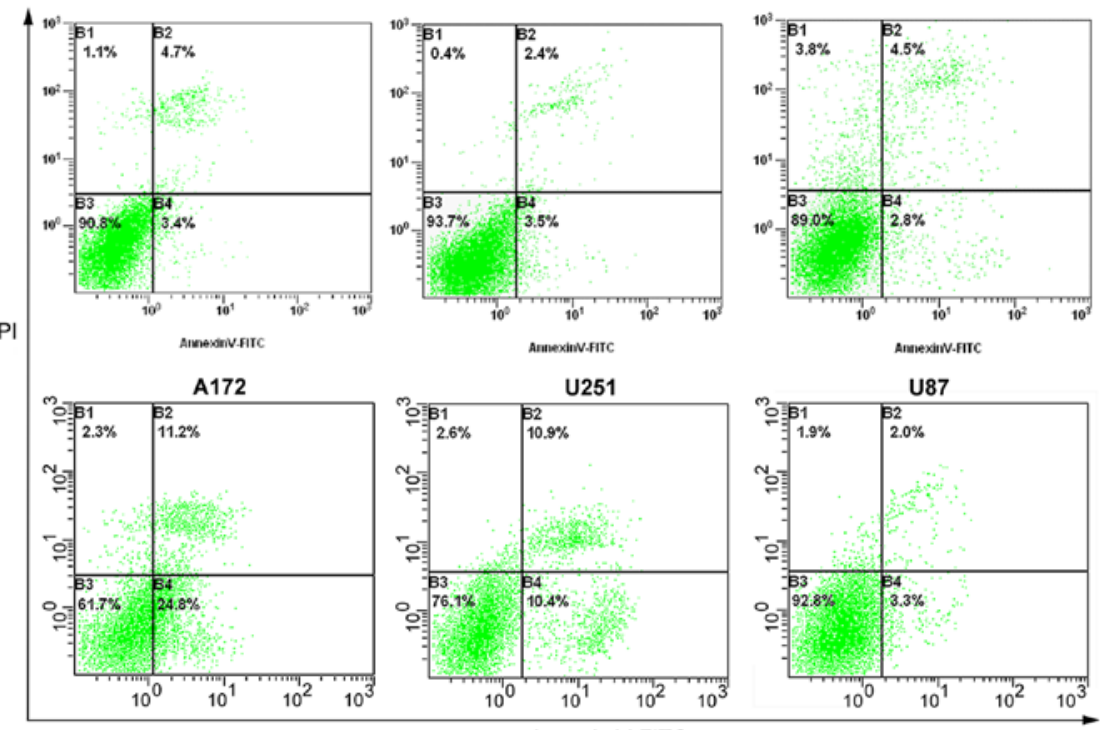

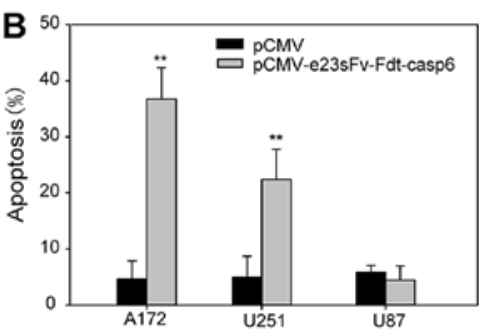

Figure 6. Induction of apoptosis in HER2-positive glioblastoma multiforme (GBM) cells in the presence of the e23sFv-Fdt-casp6 fusion protein. (A) After $48 \mathrm{~h}$ of culture in medium containing the supernatants of stably transfected Chinese hamster ovary (CHO) cells, A172, U251MG, and U87MG cells were stained with Annexin V-FITC/PI and analyzed via flow cytometry. (B) Bar graphs showing the proportion of apoptotic cells in each cell line expressed as means \pm SD from 3 independent assays. ${ }^{*} \mathrm{P}<0.05 ;{ }^{* *} \mathrm{P}<0.01$.
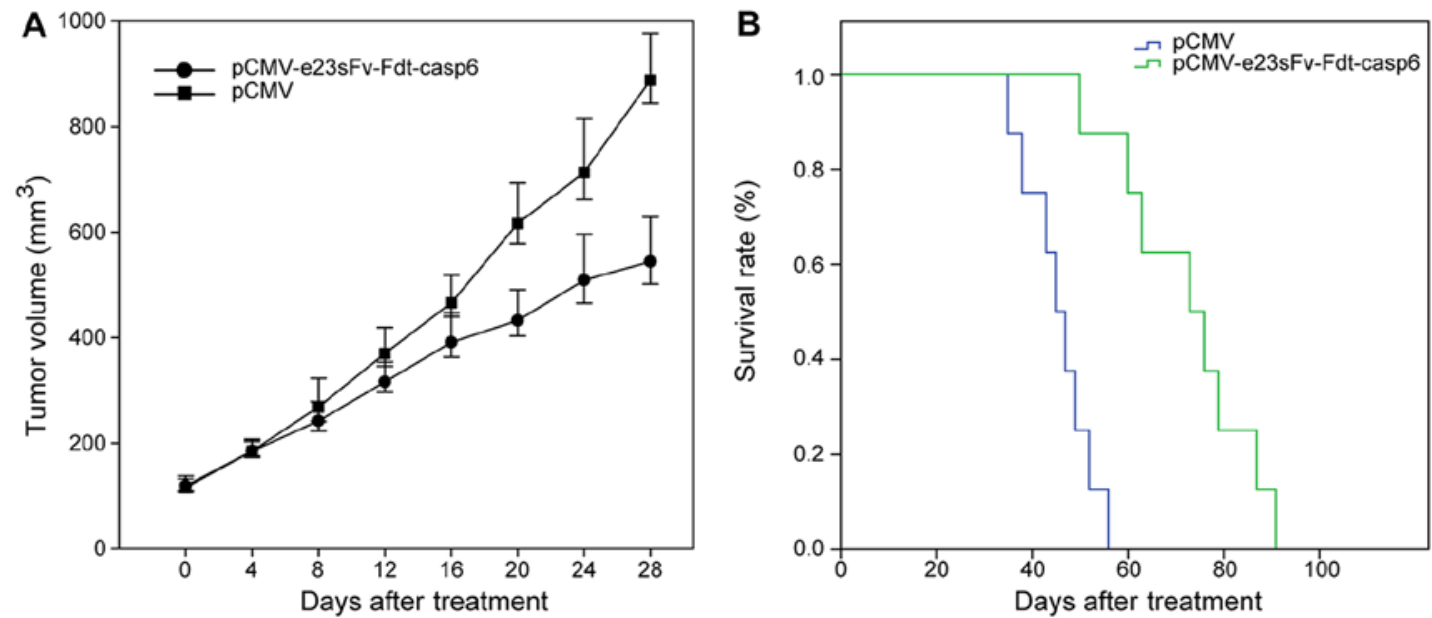

Figure 7. Antitumor activity of e23sFv-Fdt-casp6 in vivo. (A) Graph showing the change of tumor volume over time in mice injected with Lipofectamineencapsulated pCMV-e23sFv-Fdt-casp6 or the pCMV plasmid (control) intramuscularly in the right posterior limb. (B) Comparison of the survival rates for the mice bearing U251MG xenografts after treatment, as described in (A).

\section{Discussion}

Malignant gliomas, such as GBM, are the most common and lethal intracranial tumors. The standard therapy for newly diagnosed malignant gliomas involves surgical resection (when feasible), radiotherapy, and chemotherapy. Malignant gliomas cannot be eliminated completely because of their infiltrative nature, and their resistance to conventional therapies $(1,2)$. Therefore novel therapeutic strategies are necessary. The past two decades have witnessed striking advances in GBM immunotherapy. HER 2 is not expressed in the adult central nervous system, however, its expression increases with the degree of astrocytoma anaplasia. It is estimated that HER2 is expressed by up to $80 \%$ of GBM cases $(6,7)$, and therefore is ideal target 


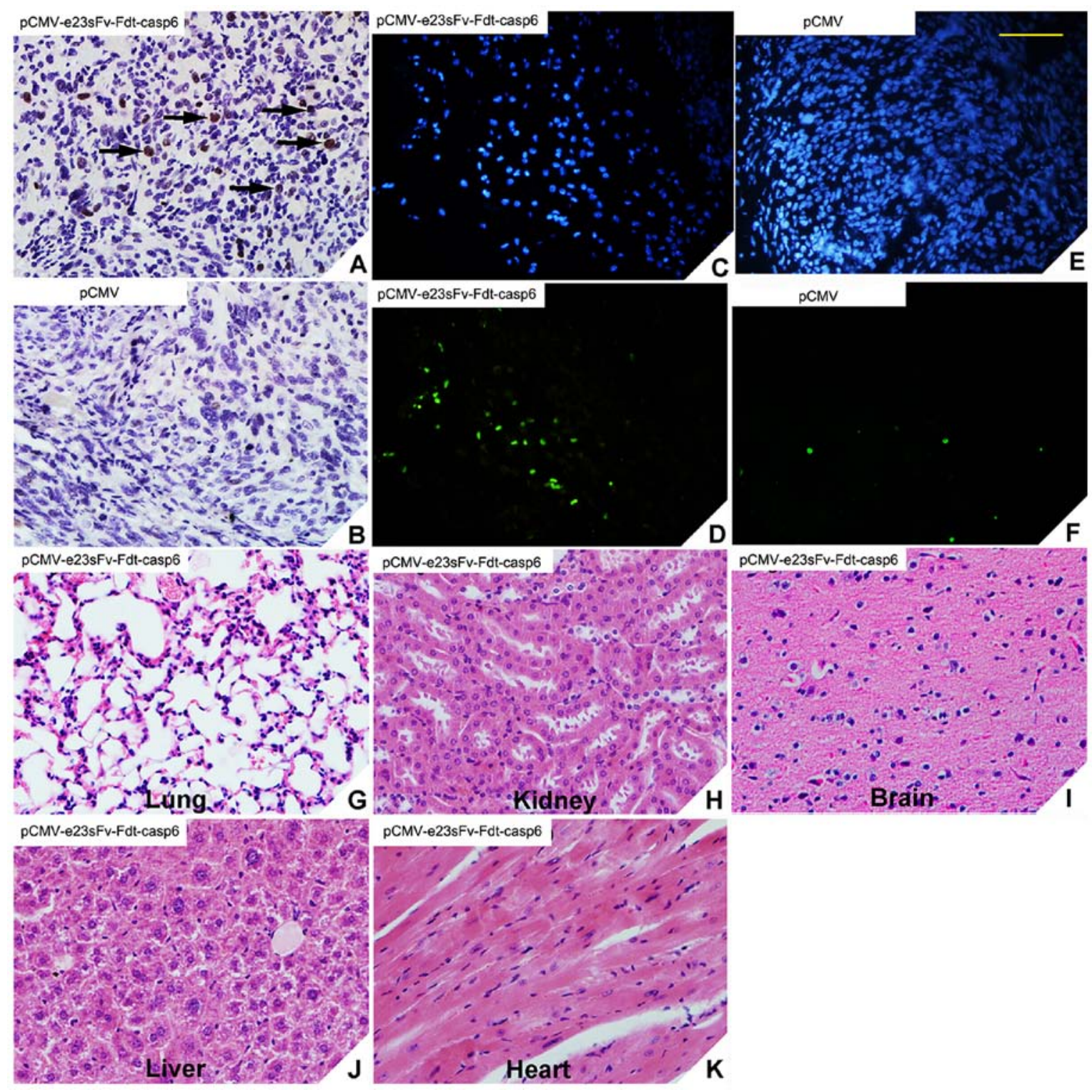

Figure 8. Immunohistochemical and histological examination of the glioblastoma multiforme (GBM) xenografts. (A and B) Distribution of e23sFv-Fdt-casp6 in tissue sections of the resected GBM xenografts detected using immunohistochemical analysis. Caspase- 6 could only be detected in xenograft sections from mice that received the Lipofectamine-encapsulated pCMV-e23sFv-Fdt-casp6 complex. The arrowheads indicate caspase-6-positive cells. (C-F) Apoptosis in the GBM xenografts in mice that received the Lipofectamine-encapsulated pCMV-e23sFv-Fdt-casp6 complex or pCMV plasmid (green). Cell nuclei were counterstained with DAPI (blue). (G-K) Hematoxylin and eosin staining of the lung, kidney, brain, liver, and heart tissue of the mice treated with the e23sFv-Fdt-casp6 construct revealed no significant indications of cell injury or damage. Magnification, $\mathrm{x} 400$.

for therapeutic approaches. Herceptin was reported to induce cellular apoptosis in GBMs overexpressing HER2 in vitro (19). In our study, the novel e23sFv-Fdt-casp6 specifically targeted HER2-overexpressing GBM cells and exhibited its killing activity in vitro and in vivo.

To investigate the specific killing activities of e23sFv-Fdt-casp6 protein, we preformed subcutaneous implantation to establish animal model in SCID mice, and the e23sFv-Fdt-casp6 gene was introduced intramuscularly to the xenograft mouse models by liposome encapsulation. Cells were transfected with the immuno-casp6 plasmid and were expected to exert their apoptosis-inducing effects in a paracrine manner. To our knowledge, one of the differences between the subcutaneous and intracranial implantations is the blood-brain barrier (BBB) that is usually very efficient in blocking most of the molecules outside the central nervous system (19). Proteins
(150 kDa), such as IgG, are thought to cross the abnormal GBM $\mathrm{BBB}$, and an intravital fluorescence microscopical approach demonstrated that tumoral microvascularization produces an abnormal BBB (20). The molecular weight of recombinant e23sFv-Fdt-casp6 protein $(67 \mathrm{kDa})$ is smaller than that of Herceptin, and is therefore likely to cross the BBB. We are conducting further experiments to validate this. In addition, subcutaneous tumors can be repetitively measured through the skin. Although intracranial implantations are more clinically relevant, the tumor sizes can be measured only when the animals are sacrificed, making it difficult to evaluate the anti-tumor activity of e23sFv-Fdt-casp6.

There are already many strategies of using HER2 as the therapy target for treating GBM, including adoptive cell therapies with chimeric antigen receptor (CAR) expressing T cells (21), activated T cells ATC armed with 
bispecific antibodies, and activated dendritic cell based immunotherapy $(22,23)$, which are already into the phase I trial. In the present study, we used a DNA based method to introduce the functional therapeutic elements into the tumor cell lines and also the animal tumor model, both were effective on successful treatment of GBM. Nucleic acid vaccines are an alternative to attenuated bacterial antigens or protein or peptide vaccines. The advantage of DNA therapeutic vaccine include: inducing humoral and cellular immune response at the same time, various promoters, enhancers, and other elements could be chosen to control the expression of the encoded protein, easy production, safer, more stable, and inexpensive (24-26). However, the major problem is that DNA vaccines may have a relatively poor immunogenicity (27). In our results, the immunogenicity is proven enough to eliminate the tumor, both in vitro to induce apoptosis, and in vivo to eliminate the tumor size in the SCID mouse model. Thus, our approach has potential to further develop to clinical use.

In conclusion, our evidence of e23sFv-Fdt-casp6's ability to induce apoptosis or cytotoxicity in HER2-overexpressing GBM cells makes it a promising therapeutic alternative for GBM treatment.

\section{Acknowledgements}

This study was supported by grants from the National Natural Science Foundation of China (no. 30872978 and no. 81301702). We thank the research staff who supported this study, including Jin-Xiang Cheng and Xiao-Liang Yang.

\section{References}

1. Wen PY and Kesari S: Malignant gliomas in adults. N Engl J Med 359: 492-507, 2008.

2. Furnari FB, Fenton T, Bachoo RM, Mukasa A, Stommel JM, Stegh A, Hahn WC, Ligon KL, Louis DN, Brennan C, et al: Malignant astrocytic glioma: Genetics, biology, and paths to treatment. Genes Dev 21: 2683-2710, 2007.

3. Ohgaki $\mathrm{H}$ and Kleihues P: Genetic pathways to primary and secondary glioblastoma. Am J Pathol 170: 1445-1453, 2007.

4. Lupu R, Colomer R, Kannan B and Lippman ME: Characterization of a growth factor that binds exclusively to the erbB-2 receptor and induces cellular responses. Proc Natl Acad Sci USA 89: 2287-2291, 1992.

5. Koka V, Potti A, Forseen SE, Pervez H, Fraiman GN, Koch M and Levitt R: Role of Her-2/neu overexpression and clinical determinants of early mortality in glioblastoma multiforme. Am J Clin Oncol 26: 332-335, 2003.

6. Ahmed N, Salsman VS, Kew Y, Shaffer D, Powell S, Zhang YJ, Grossman RG, Heslop HE and Gottschalk S: HER2-specific $\mathrm{T}$ cells target primary glioblastoma stem cells and induce regression of autologous experimental tumors. Clin Cancer Res 16: 474-485, 2010.

7. Liu G, Ying H, Zeng G, Wheeler CJ, Black KL and Yu JS: HER-2, gp100, and MAGE-1 are expressed in human glioblastoma and recognized by cytotoxic $\mathrm{T}$ cells. Cancer Res 64: 4980-4986, 2004.

8. Bian XW, Shi JQ and Liu FX: Pathologic significance of proliferative activity and oncoprotein expression in astrocytic tumors. Anal Quant Cytol Histol 22: 429-437, 2000.

9. Forseen SE, Potti A, Koka V, Koch M, Fraiman G and Levitt R: Identification and relationship of HER-2/neu overexpression to short-term mortality in primary malignant brain tumors Anticancer Res 22: 1599-1602, 2002.
10. Mineo JF, Bordron A, Quintin-Roué I, Loisel S, Ster KL, Buhé V, Lagarde N and Berthou C: Recombinant humanised anti-HER2/neu antibody (Herceptin) induces cellular death of glioblastomas. Br J Cancer 91: 1195-1199, 2004.

11. Press MF, Cordon-Cardo C and Slamon DJ: Expression of the HER-2/neu proto-oncogene in normal human adult and fetal tissues. Oncogene 5: 953-962, 1990.

12. Bold RJ, Termuhlen PM and McConkey DJ: Apoptosis, cancer and cancer therapy. Surg Oncol 6: 133-142, 1997.

13. Riedl SJ and Shi Y: Molecular mechanisms of caspase regulation during apoptosis. Nat Rev Mol Cell Biol 5: 897-907, 2004.

14. Srinivasula SM, Ahmad M, MacFarlane M, Luo Z, Huang Z, Fernandes-Alnemri T and Alnemri ES: Generation of constitutively active recombinant caspases- 3 and -6 by rearrangement of their subunits. J Biol Chem 273: 10107-10111, 1998.

15. Komata T,Kondo Y,Kanzawa T,Hirohata S, Koga S, Sumiyoshi H, Srinivasula SM, Barna BP, Germano IM, Takakura M, et al: Treatment of malignant glioma cells with the transfer of constitutively active caspase- 6 using the human telomerase catalytic subunit (human telomerase reverse transcriptase) gene promoter. Cancer Res 61: 5796-5802, 2001.

16. Ren JL, Meng YL, Hu B, Jia LT, Zhang R, Xu YM, Xie QS, Zhang YQ, Jin BQ, Chen SY, et al: The effect of direct translocation across endosomes on the cytotoxicity of the recombinant protein e23sFv-Fdt-casp6 to HER2 positive gastric cancer cells. Biomaterials 32: 7641-7650, 2011.

17. Wang T, Zhao J, Ren JL, Zhang L, Wen WH, Zhang R, Qin WW, Jia LT, Yao LB, Zhang YQ, et al: Recombinant immunoproapoptotic proteins with furin site can translocate and kill HER2-positive cancer cells. Cancer Res 67: 11830-11839, 2007.

18. Omasa T, Onitsuka M and Kim WD: Cell engineering and cultivation of chinese hamster ovary $(\mathrm{CHO})$ cells. Curr Pharm Biotechnol 11: 233-240, 2010.

19. Mineo JF, Bordron A, Quintin-Roué I, Maurage CA, Buhé V, Loisel S, Dubois F, Blond S and Berthou C: Increasing of HER 2 membranar density in human glioblastoma U251MG cell line established in a new nude mice model. J Neurooncol 76: 249-255, 2006.

20. Vajkoczy P, Schilling L, Ullrich A, Schmiedek P and Menger MD: Characterization of angiogenesis and microcirculation of high-grade glioma: An intravital multifluorescence microscopic approach in the athymic nude mouse. J Cereb Blood Flow Metab 18: 510-520, 1998.

21. Hegde M, Corder A, Chow KK, Mukherjee M, Ashoori A, Kew Y, Zhang YJ, Baskin DS, Merchant FA, Brawley VS, et al: Combinational targeting offsets antigen escape and enhances effector functions of adoptively transferred T cells in glioblastoma. Mol Ther 21: 2087-2101, 2013.

22. Akiyama Y, Oshita C, Kume A, Iizuka A, Miyata H, Komiyama M, Ashizawa T, Yagoto M, Abe Y, Mitsuya K, et al: $\alpha$-type-1 polarized dendritic cell-based vaccination in recurrent high-grade glioma: A phase I clinical trial. BMC Cancer 12: 623,2012

23. Phuphanich S, Wheeler CJ, Rudnick JD, Mazer M, Wang H, Nuño MA, Richardson JE, Fan X, Ji J, Chu RM, et al: Phase I trial of a multi-epitope-pulsed dendritic cell vaccine for patients with newly diagnosed glioblastoma. Cancer Immunol Immunother 62: 125-135, 2013.

24. Sasaki S, Takeshita F, Xin KQ, Ishii N and Okuda K: Adjuvant formulations and delivery systems for DNA vaccines. Methods 31: 243-254, 2003.

25. Robinson HL and Pertmer TM: DNA vaccines for viral infections: Basic studies and applications. Adv Virus Res 55: 1-74, 2000

26. Sun Y, Hu YH, Liu CS and Sun L: Construction and analysis of an experimental Streptococcus iniae DNA vaccine. Vaccine 28: 3905-3912, 2010

27. Kim D, Hung CF, Wu TC and Park YM: DNA vaccine with $\alpha$-galactosylceramide at prime phase enhances anti-tumor immunity after boosting with antigen-expressing dendritic cells. Vaccine 28: 7297-7305, 2010 